\title{
Il terremoto di Tolmezzo del 26 Aprile 1959
}

\author{
II PARTE
}

\section{Studio macrosismico-gelogico: determinazione analitica delle grandezze ipocentrali e natura della scossa}

\author{
P. Feliziani - L. Marcelli
}

Ricevuto il 2 Aprile 1966

Riassunto. - In questo lavoro, che costituisce la seconda parte di uno studio approfondito condotto sul terremoto avvenuto nei pressi di Tolmezzo il 26 Aprile 1959, vengono esposti e discussi i risultati dell'analisi macrosismica della scossa con relativa costruzione delle isosiste.

Vengono inoltre date delle notizie di carattere geologico, e fatti degli accostamenti.

Lo studio analitico del terremoto in esame ha ubicato l'ipocentro nel punto di coordinate

$$
\left\{\begin{array}{l}
\Phi_{0}=\left(46^{\circ} 28^{\prime} 18^{\prime \prime}, 57 \pm 2^{\prime} 42^{\prime \prime}, 17\right) \mathrm{N} \\
\Lambda_{0}=\left(13^{\circ} 00^{\prime} 44^{\prime \prime}, 16 \pm 1^{\prime} 41^{\prime \prime}, 28\right) \mathrm{E} \\
h=(5,91 \pm 10,90) \mathrm{km}
\end{array}\right.
$$

e ha dato il seguente tempo origine:

$$
H_{0}=14^{\mathrm{h}} 45^{\mathrm{m}} 14^{\mathrm{s}}, 01 \pm 0^{\mathrm{s}}, 89
$$

Si è passati indi ad esaminare la natura fisica della scossa; ne è risultata una frattura, analogamente a quanto constatato per altri terremoti ubicati nelle Alpi Orientali. Si pongono in relazione questi risultati con la geologia della zona epicentrale e si pongono in rilievo alcune caratteristiche comuni ad altri eventi sismici in detta zona.

Summary. - In this paper - the second part of a careful study on the Tomezzo earthquake of April 26th 1959 - the results of the shock macroseismic analysis and relative isoseismal lines are shown and discussed. Moreover, some geologic informations and comparisons are given. 
The hypocentral co-ordinates and the origin-time obtained by analytical study are the following:

$$
\left\{\begin{array}{l}
\Phi_{o}=\left(46^{\circ} 28^{\prime} 18^{\prime \prime}, 57 \pm 2^{\prime} 42^{\prime \prime}, 17\right) \mathrm{N} \\
A_{o}=\left(13^{\circ} 00^{\prime} 44^{\prime \prime}, 16 \pm 1^{\prime} 41^{\prime \prime}, 28\right) \mathrm{S} \\
h=(5,91 \pm 10,90) \mathrm{km} \\
H_{0}=14^{\mathrm{h}} 45^{\mathrm{m}} 14^{\mathrm{s}}, 01 \pm 0^{\mathrm{s}}, 89
\end{array}\right.
$$

It is examined the shock's mechanism: according to other Oriental Alps earthquakes, a fracture-scheme has been found.

These results are related to geological character of the epicentral zone and some characteristics - analogous to other seismic events in this zone -are remarked.

\section{RICHIMMI E GENERALITA.}

In una nota precedente abbiamo svolto una inclagine accurata sulla sismicità di quella regione dell'Italia Nord-Orientale che si estende tra il lago di Garda, l'Austria, la Jugoslavia e il Mare Adriatico. Tale indagine, che comprende tutte le notizie che si sono potute raccogliere tra l'anno 0 dell'Era Cristiana e l'anno 1963 ha messo in luce una attività particolarmente intensa e frequente in tutta la Carnia, specialmente intorno a Tolmezzo, con numerosi centri sismici (Arta, Paluzza, Enemonzo, Ampezzo, Tramonti, Cavazzo Carnico etc....) di intensità prevalente tra il VI ed il VII grado Mercalli: non manca qualche grado superiore accompagnato per lo più da periodi sismici.

L'elaborazione dei dati ha consentito altresi una visione panoramica di tutta la regione anche dal punto di vista dell'energia e della frequenza dei sismi.

Lo studio svolto in detta nota costituisce una premessa al lavoro attuale nel quale ci proponiamo lo studio completo del terremoto avvenuto nei pressi di Tolmezzo il 26 Aprile 1959, manifestatosi ivi con una intensità massima VII-VIII Mercalli.

Avendo già trattato il problema macrosismico generale della sismicità di una regione piuttosto vasta comprendente al suo interno la porzione intorno a Tolmezzo, rivolgeremo or la nostra attenzione su una zona molto più ristretta. Inizieremo con la costruzione delle isosiste del terremoto del 26 Aprile 1959: ad essa faremo seguire un breve cenno sulla geologia del territorio circostante l'epicentro. Esaminato cosi l'aspetto macrosismico-geologico del fenomeno in questione procederemo alla ricerca analitica dei dati ipocentrali, per terminare infine con una indagine sulla natura della scossa all:ipocentro. 


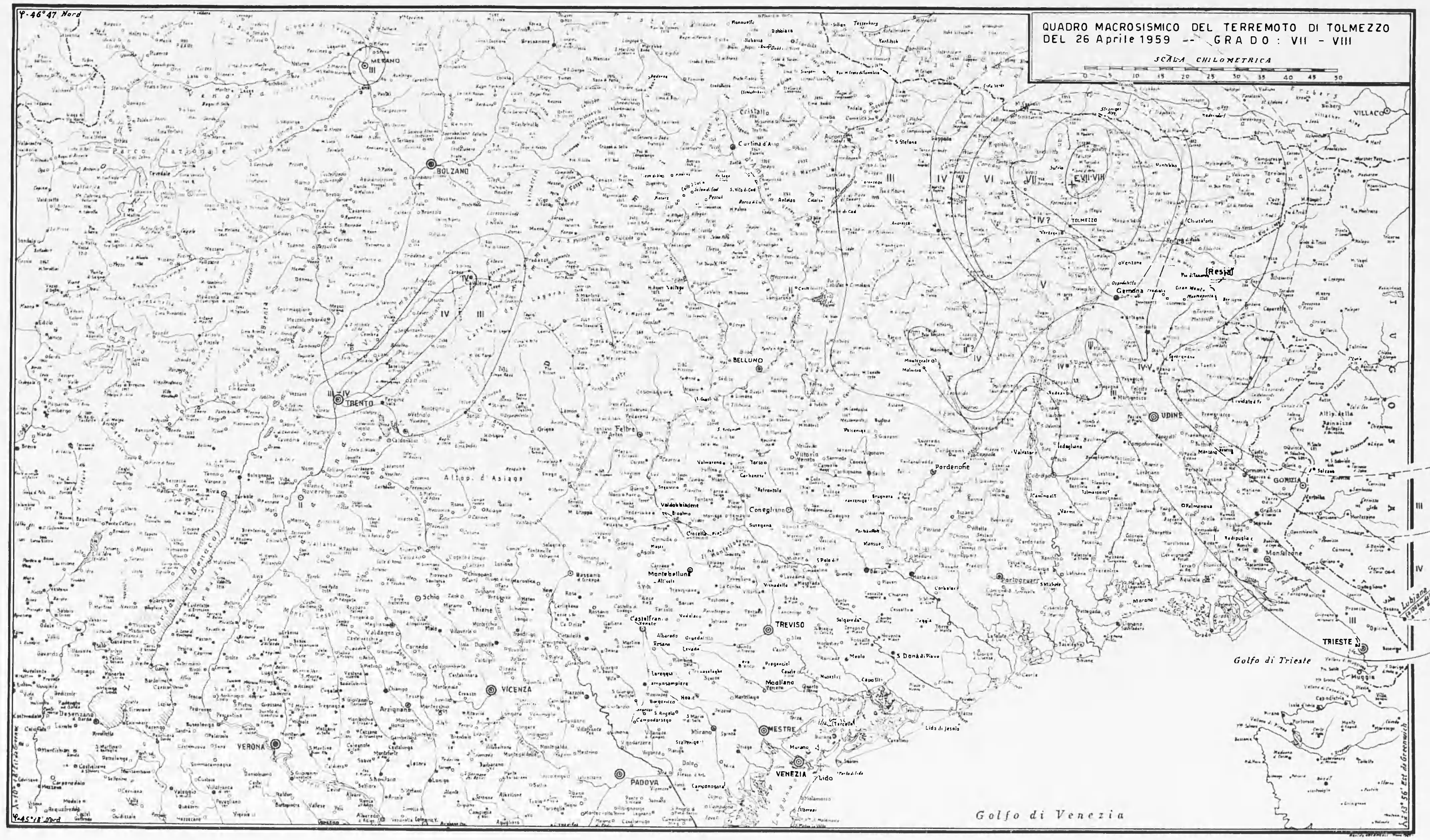


S'TUdo macrosismico deL 'TERREMo'To DEL 26 APRILe 1959 - Isosiste.

Il terremoto che il 26 Aprile 1959 , alle $15^{\mathrm{h}} 46^{\mathrm{m}}$ circa (ora italiana) scosse in maniera piuttosto violenta la zona di Tolmezzo, ebbe magnitudo 5,4 il che equivale ad uno sviluppo di energia sismica pari a 10:0 erg. ca. Esso interessò una regione abbastanza estesa dell'Italia NordOrientale, comprendente le provincie di Trieste, Udine e Belluno.

Per poter studiare macrosismicamente il terremoto, ci siamo serviti delle notizie trasmesse - dai vari paesi interessati da questo scuotimento, - al "Servizio Macrosismico» dell'Ufficio Centrale di Meteorologia.

L'esame accurato di tali notizie ci ha consentito la costruzione di una carta macrosismica (v. Fig. 1): purtroppo la mancanza di dati provenienti dai paesi stranieri lascia incompleta la documentazione. Siamo in grado tuttavia di stabilire che la massima intensità macrosismica - di VII-VIII della scala Mercalli-Sieberg — è stata raggiunta entro un'area di circa $70 \mathrm{kmq}$ a Nord di Tolmezzo, tagliando fuori da quest'area di massima intensità Tolmezzo stessa che invece è stata interessata da un VII-VI grado. L'area macrosismica, considerata fino al IIIo Mercalli si estende complessivamente per circa $12.000 \mathrm{kmq}$ : essa raggiunge ad Est la Jugoslavia, a Nord l'Austria, i Monti Cristallo; ad Ovest investe il Cadore e si smorza contro la Val di Zoldo per riprendere più in là, verso la Val di Fiemme e la Val Sugana; in direzione Sud-Est penetra con uno sperone eccezionalmente anomalo oltre il confine Jugoslavo.

La tabella I raccoglie i dati macrosismici opportunamente vagliati e ordinati. Va notato il fatto che, essendoci state nel giro di pochi minuti più scosse nella zona epicentrale (*) non è da escludere che le notizie pervenute si siano potute accavallare le une con le altre, di modo che la scossa più forte, che è quella che abbiamo studiato, può essere stata alterata nella sua prima descrizione macrosismica. La carta della Fig. 1 mette in evidenza una notevole anomalia nella propagazione dell'energia sismica in superficie e questo, ovviamente, deve esser messo in relazione con la morfologia della zona che si presenta assai tormentata. Il VII-VIII grado si distribuisce entro una area pressoché ellittica intorno al grado più elevato dell'epicentro macrosismico: quest'ultimo,

(*) Sul numero di queste scosse però non tutti i comuni che hanno fornito notizie si son trovati l'accordo. 
Tabella I

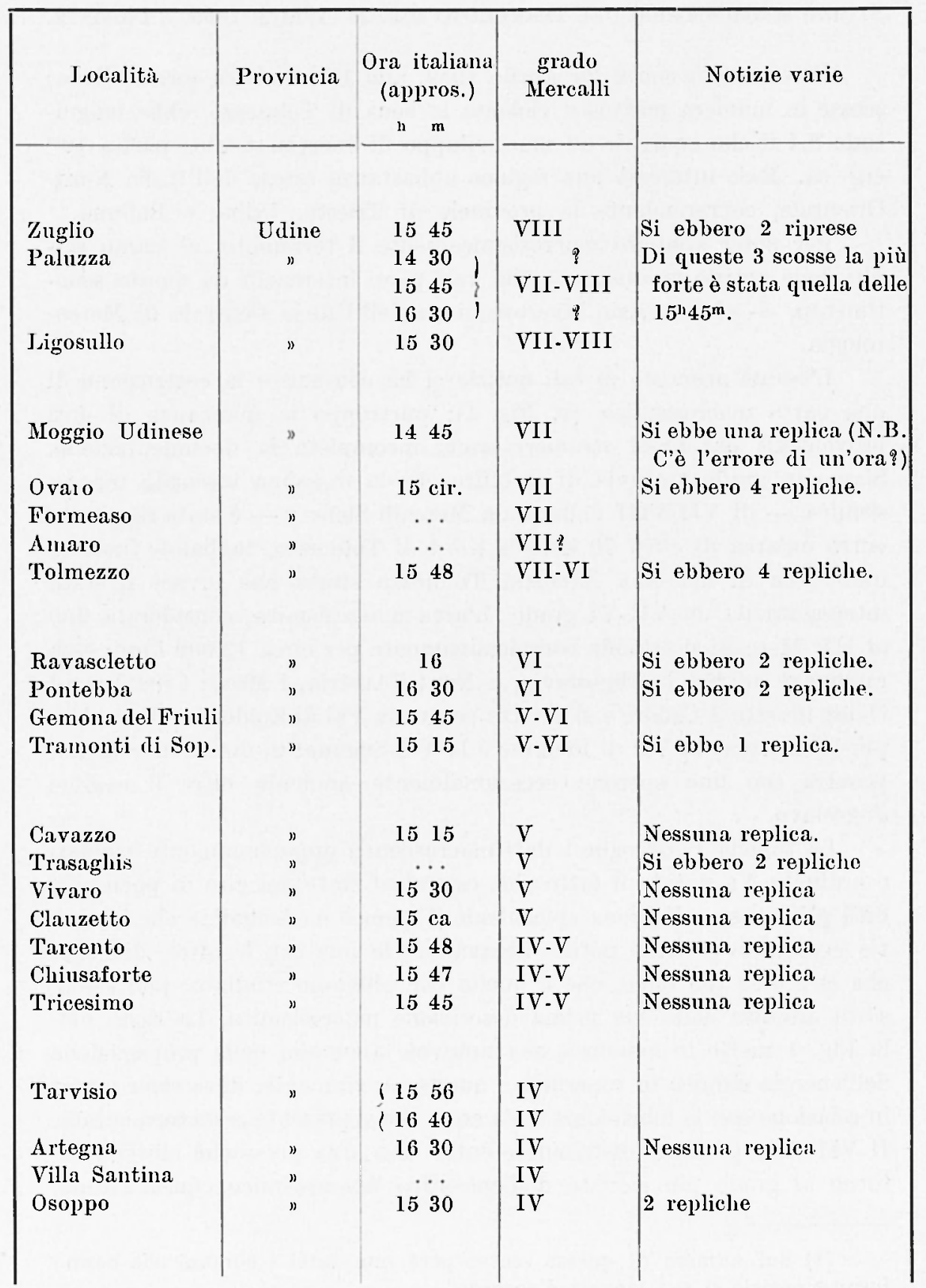


(segue) Tabella I

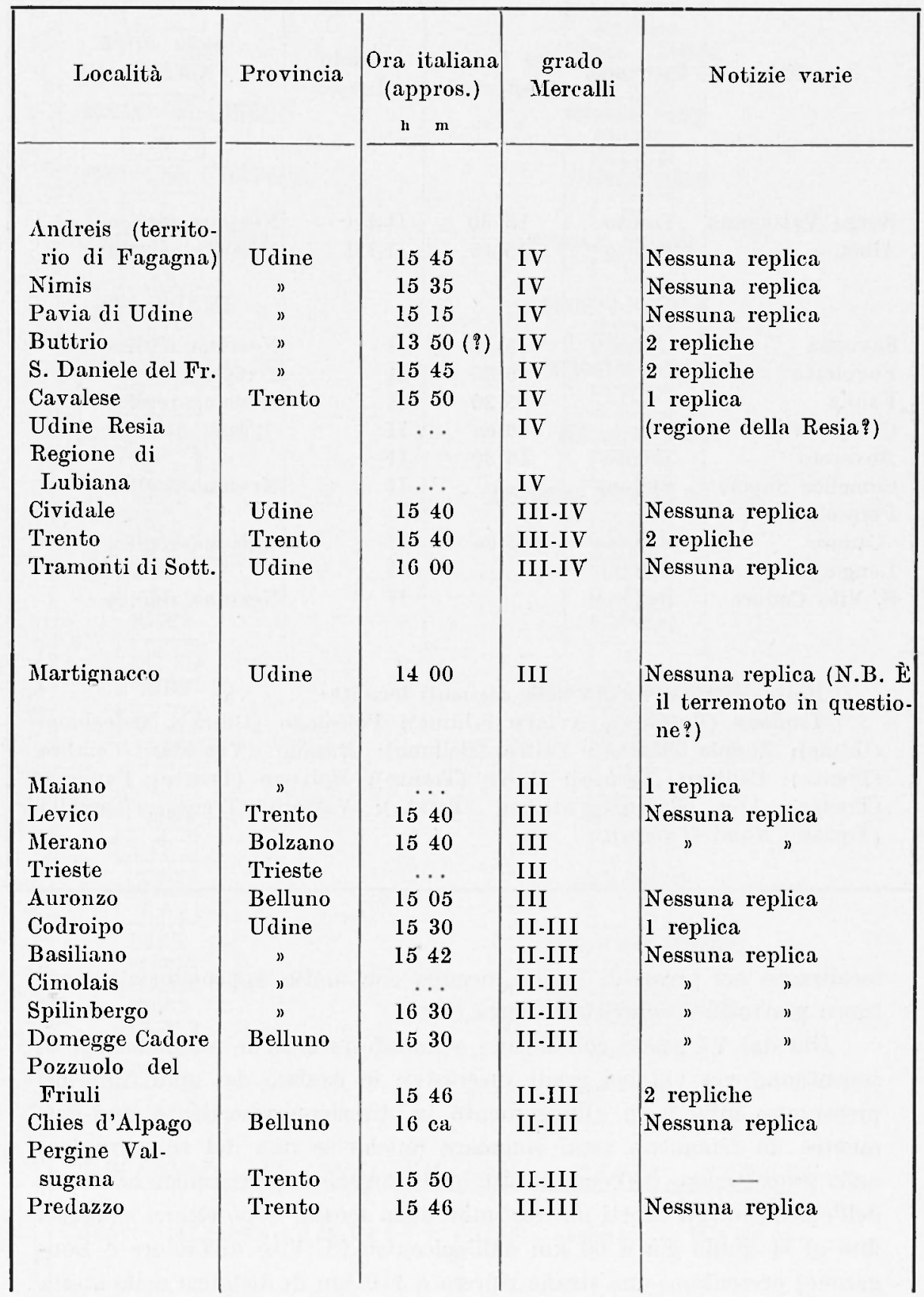


(segue) Tabella I

\begin{tabular}{|c|c|c|c|c|}
\hline Località & Provincia & $\begin{array}{c}\text { Ora italiana } \\
\text { (appros.) } \\
\mathbf{n} \mathbf{m}\end{array}$ & $\begin{array}{l}\text { Grado } \\
\text { Mercalli }\end{array}$ & Notizie varie \\
\hline $\begin{array}{l}\text { Borgo Valsugana } \\
\text { Albiano }\end{array}$ & $\begin{array}{l}\text { Trento } \\
\text { Trento }\end{array}$ & $\begin{array}{ll}15 & 30 \\
15 & 45\end{array}$ & $\begin{array}{l}\text { II-III } \\
\text { II-III }\end{array}$ & $\begin{array}{ll}\text { Nessuna } & \text { replica } \\
\text { Nessuna } & \text { replica }\end{array}$ \\
\hline $\begin{array}{l}\text { Savogna } \\
\text { Povoletto } \\
\text { Famna } \\
\text { Campoformido } \\
\text { Rovereto } \\
\text { Comelico Super. } \\
\text { Perarolo di } \\
\text { Cadore } \\
\text { Longarone } \\
\text { S. Vito Cadore }\end{array}$ & 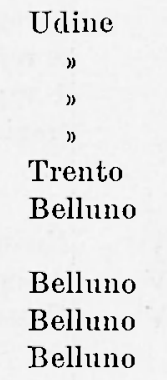 & $\begin{array}{cc}15 & 45 \\
15 & 35 \\
15 & 30 \\
15 & \mathrm{ca} \\
15 & 30 \\
\ldots \\
15 \mathrm{ca} \\
\ldots \\
\ldots\end{array}$ & $\begin{array}{l}\text { II } \\
\text { II } \\
\text { II } \\
\text { II } \\
\text { II } \\
\text { II } \\
\\
11 \\
\text { II } \\
\text { II }\end{array}$ & $\begin{array}{l}\text { Nessuna replica } \\
2 \text { repliche } \\
\text { Nessuna replica } \\
\text { Nessuma replica } \\
\text { Nessuna replica } \\
\text { Nessuna replica }\end{array}$ \\
\hline $\begin{array}{r}\text { Non è stat } \\
\text { Trodena ( } \\
\text { (Udine); Zoffola } \\
\text { (Trento); Callia } \\
\text { (Trento); Arco } \\
\text { ('Trento); Nomi }\end{array}$ & $\begin{array}{l}\text { avvertita } \\
\text { (Uolzano); A } \\
\text { (Udine); } \\
\text { (To (Trento) } \\
\text { (Trento); } \\
\text { (Trento). }\end{array}$ & $\begin{array}{l}\text { nelle seguenti } \\
\text { viano (Udine } \\
\text { Feltre (Bellu } \\
\text {; Giovo (Tre } \\
\text { Strigno (Tres }\end{array}$ & $\begin{array}{l}\text { località: } \\
\text { ); Polcenis } \\
\text { no); Bura } \\
\text { nto); Molv } \\
\text { to); Vatt }\end{array}$ & $\begin{array}{l}\text { o (Udine); Sedegliano } \\
\text { o (Venezia); Cembra } \\
\text { no (Trento); Pannone } \\
\text { ro ('Trento); Tassullo }\end{array}$ \\
\hline
\end{tabular}

localizzato nei pressi di Zuglio, occupa con molta approssimazione un fuoco piuttosto eccentrico di detta ellisse.

Già dal VI grado cominciano a manifestarsi delle anomalie che si accentuano via via nei gradi successivi: le isosiste dei gradi inferiori presentano infatti un allungamento in direzione prevalente Sud-Est, mentre un fenomeno assai singolare (anche se non del tutto nuovo) nella propagazione dell'energia sismica in superficie, si riscontra ad Ovest dell'epicentro: gli effetti macrosismici della scossa, dopo essersi smorzati fino al II grado già a $60 \mathrm{~km}$ dall'epicentro (S. Vito di Carore e Longarone) presentano una strana ripresa a $110 \mathrm{~km}$ di distanza nella stessa 


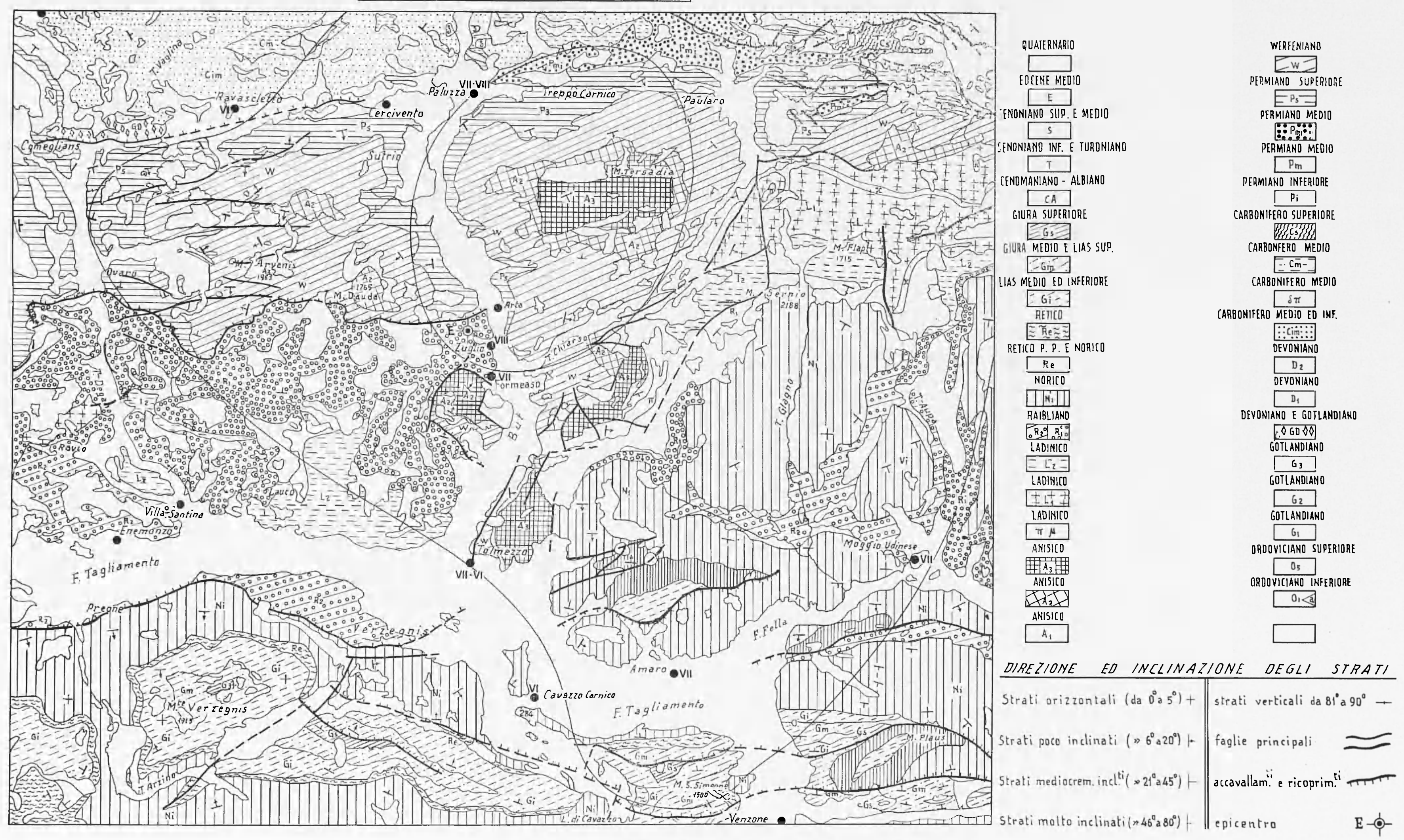




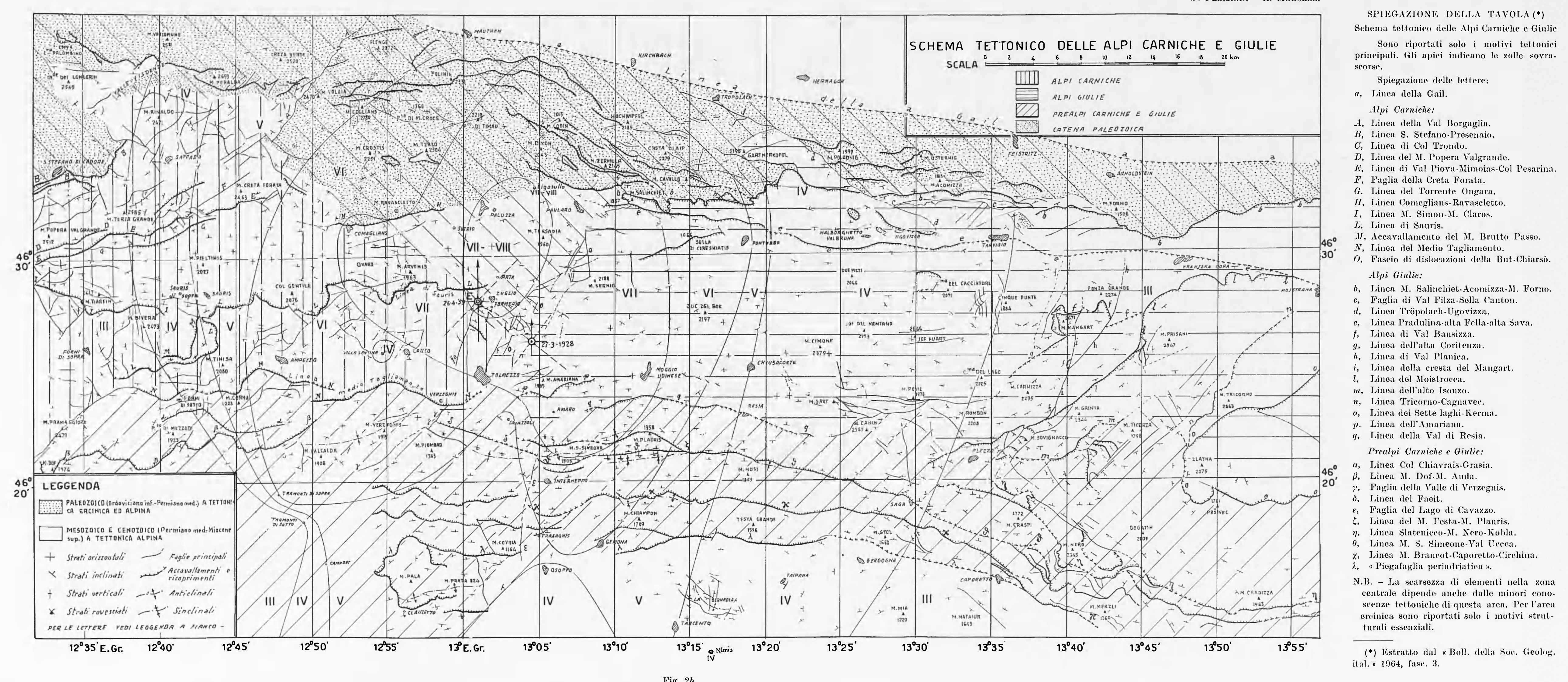


direzione con il II-III di Predazzo, che addirittura si esalta nel IV di Cavalese $10 \mathrm{~km}$ ancora più distante. Il III grado di Merano - isolato a più di $150 \mathrm{~km}$ dall'epicentro - rende ancora più evidente lo strano comportamento della zona in fatto di propagazione elastica.

Già nello studio fatto dal geologo Gortani su "Il terremoto del 27 Marzo 1928 nelle prealpi dell'Arzino (Friuli) ", il cui epicentro si trova a meno di $6 \mathrm{~km}$ da quello del terremoto oggetto del nostro studio, l'autore, che ebbe modo di svolgere una accuratissima indagine con sopraluoghi diretti ebbe a notare che quello scuotimento (che raggiumse il $\mathbf{X}$ all'epicentro) aveva avuto intensità diverse anche in punti tra loro vicinissimi.

Riteniamo opportuno tratteggiare a grandi linee l'aspetto geologico della zona epicentrale per avere la possibilità di considerare parallelamente gli effetti macrosismici e la geologia del terreno.

\section{CenNi SUlla geologia DELla Regione E CONSIDERAzioni varie.}

Ci limiteremo ovviamente a quella porzione di territorio che fu maggiormente colpita dal nostro terremoto.

Tale porzione comprende una parte delle Alpi Carniche, una parte delle Alpi Giulie occidentali e la parte settentrionale delle Prealpi Carniche, considerando come limite fra le prime due il fascio di dislocazioni della linea But-Chiarsò (che va da Tolmezzo fino a Sud-Est del Monte Paularo) e ponendo la terza a Sud del Tagliamento e della Val di Resia.

Per illustrare quanto diremo ci serviremo dei due schemi geologici di Figura $2 a$ e $2 b$, il primo dei quali mette in particolare evidenza la stratigrafia della zona e il secondo la tettonica. Lo schema geologico della Fig. $2 a$ è stato costruito utilizzando come base fondamentale una parte della carta geologica delle tre Venezie (fogli di Ampezzo, Pontebba e Tarvisio) rilevata da vari geologi italiani, completata con i rilevamenti condotti dall'Istituto Geologico di Bologna in questi ultimi anni.

Il secondo (Fig. 2b) è quello che accompagna la nota del Selli pubblicata sul Bollettino della Soc. Geolog. Italiana del 1964. Di detta nota ci serviremo prevalentemente per esaminare la geologia della zona.

L'area che ci interessa in modo particolare presenta, in così breve spazio, una gran varietà di fenomeni geologici. Dal punto di vista stratigrafico, vi compare per intero tutta la serie dell'Ordoviciano inferiore all'Eocene Medio, per uno spessore complessivo di $14-15.000 \mathrm{~m}$. circal. 
Dal punto di vista tettonico essa offre un aspetto estremamente complesso dato che è stata interessata da due grandi cicli orogenetici: l'Ercinico e l'Alpino. Vi si distinguono quattro grandi unità geologiche:

1) la catena paleocarnica dove affiora il paleozoico antico.

2) le Alpi Carniche vere e proprie, a Sud della precedente catena, delimitate ad oriente dal torrente Chiarsò, da Paularo e dal basso corso della But: il corso del Tagliamento le limita a Sud.

3) le Alpi Giulie in senso lato che si svolgono ad oriente del suddetto limite But-Chiarsò, a Sud del Paleozoico e a Nord della Val di Resia.

4) le Prealpi Carniche, a Sud del Tagliamento e della Val di Resia.

Durante il Giurese, il Cretaceo e l'Eocene, le regioni carniche e gitulie furono soggette a varie e disformi oscillazioni verticali, in relazione alle fasi del corrugamento alpino: tali oscillazioni sono denunciate da variazioni di facies $\left(^{*}\right)$ e da lacune stratigrafiche.

Agli effetti di uno studio sismologico, ciò che offre più interesse è però l'aspetto tettonico.

Sia l'orogenesi ercinica che l'alpina si sono svolte secondo un enorme numero di fasi diastrofiche (**) che non hanno avuto sempre sollecitazioni isorientate, differendo anzi spesso nettamente tra di loro per senso e direzioni delle spinte o dei movimenti risultanti. Le strutture erciniche sono state sovente deformate o addirittura cancellate dalla successiva orogenesi alpina.

Difatti, mentre in un piano tettonico più profondo - che interessa il paleozoico antico e il carbonifero inferiore e medio - si riscontrano strutture sminuzzate e sconvolte dovute a movimenti ercinici ed alpini, un IIo piano ad esso sovrapposto presenta una tettonica meno disordinata, anch'essa dovuta a movimenti tardo-ercinici ed alpini. Un IIIo piano tettonico infine, sovrapposto ai primi due e che interessa tutti i terreni dalla Val Gardena in poi, è il risultato dei soli movimenti alpini,

(*) "Facies" ò in geologia l'insieme dei caratteri tipici che riflettono le condizioni genetiche di una roccia o di una formazione.

$\left({ }^{* *}\right)$ "Diastrofismo » è il complesso delle deformazioni e delle dislocazioni subite dagli strati rocciosi dopo la loro formazione. 
ed ivi la tettonica è molto più regolare. Tuttavia anche qui, in alcune aree, compare un certo disordine strutturale che fa pensare a dei fenomeni di tettonica passiva gravitativa.

Sicchè in un breve quadro riassuntivo potremo dire che la regione in esame, oltre ad aver subito una tettonica attiva crostale, determinata da sospingimenti o compressioni delle masse o da trazioni da parte del substrato profondo, è stata oggetto anche di una chiara tettonica passiva (epidermale) di scivolamento gravitativo. Tutti questi fenomeni furono poi preceduti, accompagnati o seguiti da una subsidenza intensa, ma disforme nel tempo, o da oscillazioni di riequilibrio isostatico, che indubbiamente ebbero un'importanza notevole nell'evoluzione strutturale. Quindi è evidente che i maggiori lineamenti strutturali sono il risultato non di un solo, ma di numerosi fenomeni dinamici succedutisi variamente nel tempo.

Il territorio più fortemente colpito dal terremoto del 26 Aprile 1959 comprende, come è stato già accennato precedentemente, una parte delle Alpi Carniche, una parte delle Alpi Giulie occidentali ed una parte delle Prealpi Carniche.

Le principali linee di dislocazione e motivi tettonici che ivi si riscontrano possono essere schematizzate nel modo che segue:

I. - Linea Comeglians-Ravascletto, ( $H$ dello schema $2 b$ ) orientata $\mathrm{E}-\mathrm{W}$, che porta i terreni paleozoici su quelli permiani con un accavallamento verso $\mathrm{S}$.

II. - Sistema di dislocazioni dell'Arvenis. Sono faglie ad andamento E-W parallele alla precedente e alla seguente con piano subverticale e leggermente immerso verso $\mathrm{N}$, accompagnate da altre secondarie orientate $\mathrm{N}$-S.

III. - Linea di Sauris, ( $L$ dello schema $2 b)$ : è forse questo il motivo più esteso e cospicuo delle Alpi Carniche che si segue procedendo da $\mathrm{E}$ verso $\mathrm{W}$, dal But (presso Zuglio) al margine meridionale del Monte Rancolin (presso Forni di Sopra) con un andamento all'incirca E-W, ma molto irregolare. Essa porta il Werfeniano ed il Permiano superiore ad accavallarsi verso $\mathrm{S}$ con il Raibliano, secondo un piano debolmente inclinato verso $\mathrm{N}$. (vedi schema $2 a$ ). L'epicentro del nostro terremoto, come vedremo a calcoli fatti, risulta ubicato tra l'estremità di questa linea e la faglia " $f a$ " che quasi si immerge in essa, provenendo da SudOvest. 
IV. - Linea del Medio Tagliamento. ( $N$ dello schema $2 b$ ) Si indica così l'accavallamento del Raibliano sopra la Dolomia principale mediante una superficie fortemente immergente verso $\mathrm{N}$ e orientata $\mathrm{EW}$ che si osserva a destra della Valle fra Socchieve e la confluenza ButTagliamento. (v. Figg. $2 a$ e $2 b$ ).

V. - Nella porzione compresa fra il Tagliamento a $\mathrm{S}$ e la linea di Sauris a N, i terreni triassici, e sopratutto quelli raibliani, sono inreressati da una serie numerosa di faglie - una della quali, tra le più evidenti, è quella " $f a$ " di cui sopra — faglie inverse e piccoli locali accavallamenti. Gli orientamenti strutturali sono quanto mai vari. E proprio in uno dei punti più disordinati di questo territorio che si nota l'anomalia di Villa Santina, posta all'incrocio di due faglie. Questa località, che fa parte geograficamente di una regione molto ampia in cui il terremoto è stato avvertito di VI grado, è stata interessata soltanto da un IV grado. Anche il Gortani riscontrò un comportamento piuttosto anomalo di questa località; egli ebbe infatti a dire che l'Altipiano di Lauco (a N-E di Villa Santina) e il Monte di Verzegnis (a S-E) si comportarono, in occasione del terremoto del 1928, come massicci resistenti giacchè "soffersero un minore scuotimento" rispetto alle aree circostanti.

VI. - Particolare attenzione va posta inoltre al Fascio di dislocazioni della But-Chiarsò. (O dello schema $2 b$ ). Le Carniche al loro contatto con le Giulie fra Paularo e Tolmezzo, sono interessate da un complicato sistema di dislocazioni a prevalente orientamento NE-SW, che in certi casi presentano indizi di accavallamenti verso $\mathrm{NW}$. Ad esse si associano faglie minori con orientamenti vari. La fascia But-Chiarsò a pochi $\mathrm{km}$ dal nostro epicentro, assume geologicamente una grandissima importanza: essa rappresenta praticamente la fascia di separazione tra le Alpi Carniche e le Alpi Giulie le quali sono profondamente diverse le une dalle altre.

Infatti le Alpi Giulie sono caratterizzate in superficie dal predominante affioramento dei terreni del Trias medio e superiore con una enorme pila calcareo-dolomitica dello spessore di $3.500 \mathrm{~m}$ : i terreni sono prevalentemente rigidi e la tettonica si presenta per lo più disgiuntiva secondo grandi piani di accavallamento che hanno permesso il sovrascorrimento di enormi zolle, per prevalenti compressioni da Nord a da Sud: tali accavallamenti e sovrascorrimenti corrispondono in realtà a dei sottoscorrimenti delle zolle più esterne sotto quelle più interne. 
Le Alpi Carniche invece presentano un molto maggiore spessore sia della successione stratigrafica che dei termini plastici: questi ultimi inoltre presentano una tettonizzazione talora intensissima: le masse lungo le maggiori linee tettoniche presentano un sovrascorrimento verso Sud (al contrario delle Alpi Giulie contigue i cui forti accavallamenti si sono verificati verso Nord).

Vale la pena di insistere ancora un poco su queste considerazioni geologiche giacchè è evidente che anche se non si può esprimere in termini matematici la relazione intercorrente tra struttura geologica di una zona e manifestazioni sismiche (con relativi effetti macrosismici), pure i due fenomeni sono intimamente connessi, specie quando $\mathrm{i}$ fenomeni sismici sono di carattere tettonico.

L'interpretazione che il geologo R. Selli fa dello stile tettonico delle Alpi Carniche è quello di una serie di scollamenti e scivolamenti gravitativi, da $\mathrm{N}$ verso $\mathrm{S}$, di masse scompaginate a causa forse della intensa subsidenza del bacino carnico e della maggiore plasticità dei terreni; a queste cause va aggiunta la progressiva migrazione da $N$ verso $S$ dell'asse subsidente E-W del Bacino dal Permiano al Neocene ed infine il contemporaneo sollevamento delle aree più settentrionali. Non sono mancate, anche nelle Alpi Carniche, vere compressioni bilaterali analoghe a quelle delle Alpi Giulie.

La fascia But-Chiarsò, già predeterminata fin dal Permiano avrebbe permesso il distacco e il diverso comportamento tettonico fra i due sistemi Alpini.

Proseguendo ancora nella messa in evidenza delle principali linee di dislocazione che interessano l'area megasismica del nostro terremoto, dobbiamo parlare della:

VIT. - Linea dell'Amariana (linea " $p$ " dello schema) la quale però si trova già nelle Alpi Giulie, quasi al contatto con le prealpi. Essa è un accavallamento verso $\mathrm{N}$ del Dachstein sul Lias o sull'Eocene con piano fortemonte inclinato verso Sud. Incerto è il suo proseguimento verso $\mathrm{E}$.

VIII. - La linea della Val di Resia (" $q$ " dello schema 2b) sita fur lo sbocco della Val di Resia ed il bivio per la Carnia, ha il netto carattere di un accavallamento verso $\mathrm{N}$ del Raibliano sull'Eocene e sul Dachstein. Probabilmente si prolunga entro la Val di Resia. Essa rappresenta, in un certo senso, insieme con la linea del medio Tagliamento, la divisione tra le alpi Giulie e Carniche e le Prealpi. 
Infatti a sud di queste due linee corre la catena settentrionale delle Prealpi Carniche, costituite da una serie di scaglie tettoniche (*) allungatissime accavallate verso Sud.

Fra i motivi principali che qui interessano possiamo citare:

IX. - La sinclinale di Val Lavaruzza, tagliata a $\mathrm{S}$ da un accavallamento che interessa anche il margine settentrionale del monte $\mathrm{S}$. Simeone e confluisce ad E nella linea di Val Uccea.

X. - L'anticlinale del Monte S. Simeone, in terreni giurassici, stretta, compressa, locale e solcata sui fianchi da accavallamenti vergenti a Sud.

XI. - La linea S. Simeone-Val Uccea. ( $\theta$ » dello schema) è un grande accavallamento seguibile dal Lago di Cavazzo all'Isonzo, mediante il quale la Dolomia principale si accavalla sui terreni giuresi-cretacei.

XII. - La fagtia del Lago di Cavazzo (" $\varepsilon$ » dello schema) è un motivo trasversale ad orientamento tra N-S e NNE-SSW, che interrompe ad occidente $\mathrm{i}$ motivi strutturali precedenti.

XIII. - La faglia della Valle di Verzegnis, (“ $\gamma$ » dello schema) ad andamento NE-SW è forse in relazione con la fascia But-Chiarsò a $\mathrm{N}$ del Tagliamento.

XIV. - e infine la sinclinale Valcalda-Verzegnis, relativamente larga, breve e dolcissima, orientata ENE-WSW.

Il nostro epicentro si è trovato, grazie alle ricerche che si sono fatte e che vedremo in seguito, ad avere le seguenti coordinate $\Phi_{o}=46^{\circ} 28^{\prime}, 3 \mathrm{~N}$ e $\Lambda_{o}=13^{\circ} 00^{\prime}, 7 \mathrm{E}$.

Esse individuano un punto, nei pressi di Zuglio, situato nel pieno di una zona che è stata anche per l'addietro teatro di numerosi eventi sismici più o meno della stessa intensità (v. la Fig. 3; carta degli epicentri pubblicata anche nella I parte di questo studio) ivi compreso il grande terremoto del 27 Marzo 1928, già da noi ricordato.

$(*)$ "Scaglie tettoniche " somo, in stratigrafia, particolari strutture tettoniche dovute a modica traslazione di serie rocciose su altre preesistenti. 
P. Feliziani - L. Mircet

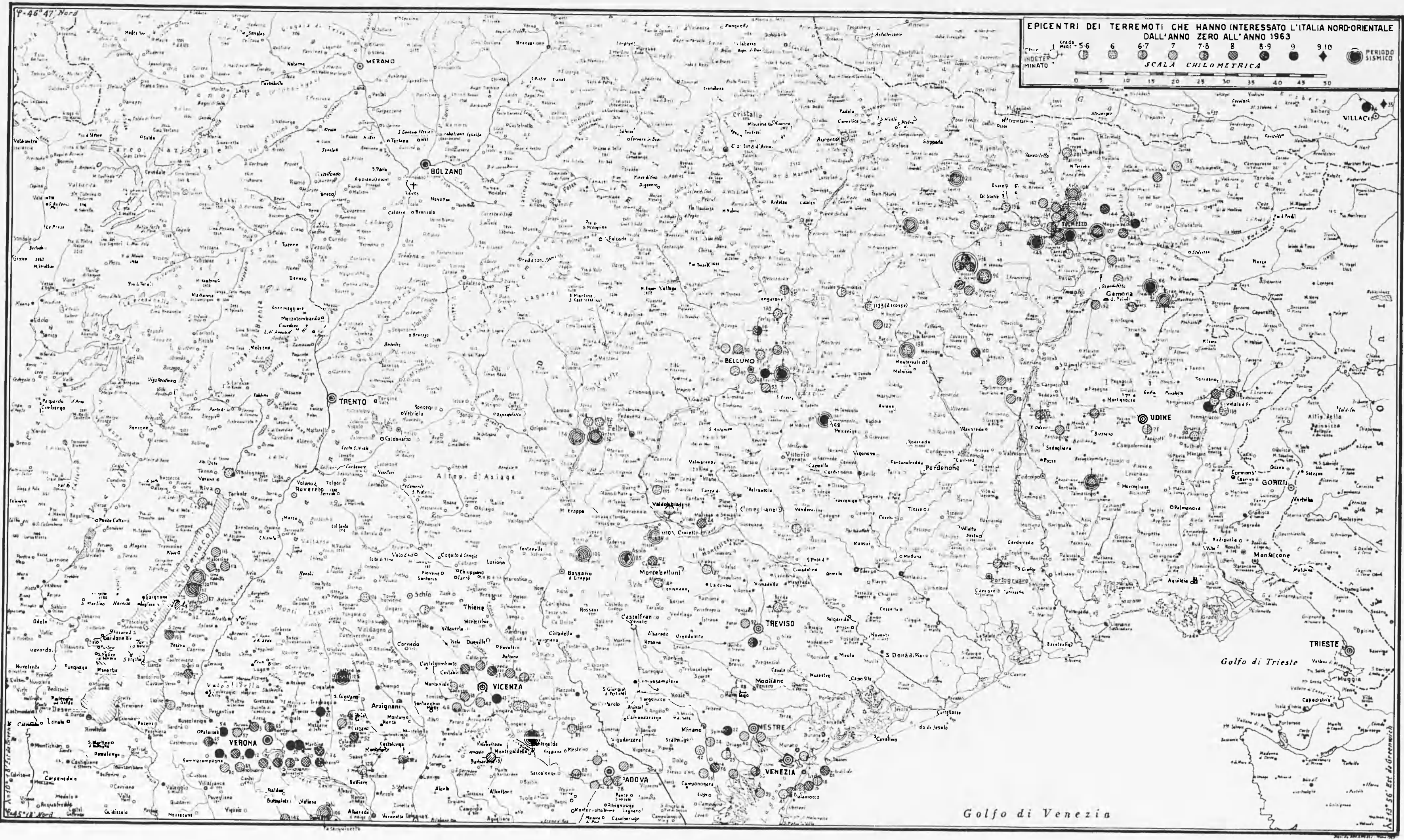


Quest'ultimo, accuratamente studiato dal prof. Gortani, era stato ubicato dall'autore $405 \mathrm{~km}$ a Sud-Est di Tolmezzo, tra il Monte S. Simeone e la faglia di Verzegnis. Avendo però lo studioso tratto tali conclusioni in base ai soli dati macrosismico-geologici, non fa meraviglia se in seguito allo studio analitico effettuato successivamente da altri autori, tale epicentro sia stato ubicato a $\mathrm{N}-\mathrm{E}$ di Tolmezzo, sulla fascia But-Chiarso, a circa $5 \mathrm{~km}$ dal nostro (v. Fig. 2b).

Non è improbabile che l'uno e l'altro trovino la loro causa nel grande e continuo movimento orogenetico che anima tuttora il grandioso complesso delle nostre Alpi.

\section{CRITICHE ED OSSERVAZIONI VARIE.}

Benchè gli studi geologici effettuati da insigni studiosi sulla regione che a noi interessa siano accurati e minuziosi e benchè in essi si possa trovare ampia materia per deduzioni sulle cause della attività sismica che anima la zona, pure non sono sufficienti da soli ad offrire una giustificazione all'andamento delle isosiste. Gli elementi che giocano sulla propagazione dell'energia sismica sono senza dubbio legati prevalentemente ai fattori geologici, ma debbono altresì dipendere da altri fattori meno evidenti e meno controllabili quale potrebbe essere, ad esempio, il modo con cui si manifesta la rottura degli equilibri interni, la profondità a cui questo avviene, il volume di terreno interessato nella zona ipocentrale, l'energia ivi sviluppata, e così via. Talchè si può ritenere che anche se ci si sforza di costruire un modello ideale del fenomeno, ben difficilmente la realtà risulta aderente alla teoria.

Del resto, se diamo un'occhiata alle numerose carte macrosismiche che illustrano la prima parte di questa nota, troviamo conferma di quanto detto circa la imprevedibilità del modo di propagarsi dell'energia sismica in superficie.

Dei 14 terremoti documentati graficamente soltanto quello del 13 Giugno 1959 (con epicentro maerosismico tra Sappada e Forni -VII-) presenta una qual certa analogia con questo che stiamo studiando; alcuni altri, con epicentro prossimo a questi, hanno andamento completamente diverso anche tra loro: unici ad essere simili, i due sismi del 10 Febbraio 1957 presso Tolmezzo $\left(\mathrm{I}_{\mathrm{o}} \mathrm{max} .=\mathrm{V}\right)$ e del 18 Febbraio 1957 presso Paluzza $\left(\mathrm{I}_{\mathrm{o}} \max .=\mathrm{V}\right)$.

Per onestà scientifica è tuttavia da tener presente anche il fatto che gli elementi che in generale si usano per la costruzione delle isosiste 
sono ancora estremamente empirici, scarsi ed inadeguati per affrontare uno studio del genere in maniera rigorosamente scientifica.

Il problema è notevole e non abbiamo conoscenza che alcuno lo abbia ancora risolto in maniera soddisfacente.

Tuttavia ogni studio, anche se imperfetto, purchè condotto con onestà e scrupolo, porta un piccolo contributo ad una conoscenza sempre più profonda e sempre meno incompleta.

Vedremo più avanti ulteriori interessanti considerazioni sull'argomento.

STUdio ANALITICO DEL TERREMOTO DEL 26 A DRILE 1959.

Procediamo ora alla impostazione analitica del problema.

E noto che qualunque studio si voglia intraprendere con un terremoto, la prima indagine va rivolta ad individuare con matematica esattezza le grandezze ipocentrali del sisma. E altresì noto che l'impresa è piuttosto complessa giacchè occorre partire da dati sperimentali che offrano serie garanzie di attendibilità.

Dopo una prima accuratissima selezione del materiale disponibile si procede all'impostazione del problema e indi alla elaborazione matematica dei dati.

\section{a) Spoglio dei sismogrammi.}

Nel nostro problema specifico, abbiamo quindi incominciato con una selezione rigorosa dei dati iniziali, scegliendo, tra i numerosi sismogrammi delle stazioni sismiche europee, quelli che maggiormente si adattavano al nostro scopo, sia per la ubicazione degli osservatori rispetto all'epicentro, sia per la garanzia offerta da requisiti strumentali. A selezione ultimata abbiamo visto che potevamo contare su una ventina di stazioni utilizzabili per gli scopi del nostro lavoro.

La stazione di Tolmezzo, vicinissima all'epicentro, avrebbe offerto delle splendide registrazioni le quali malauguratamente sono risultate inservibili perchè l'inizio è capitato proprio nell'interruzione del minuto: questo inconveniente, che per una stazione lontana avrebbe potuto risultare meno grave, ha portato invece all'esclusione di dati estremamente preziosi. 
b) Ricerca dei dati ipocentrali - Esposizione del metodo usato - Calcoli Risultati.

È noto che la ricerca analitica dei 4 dati ipocentrali $\left(\Phi_{o}, \Lambda_{o}, h, H\right)$ si può effettuare con vari metodi. Noi useremo quello dovuto a CaloiPeronaci il quale è stato applicato dagli autori e da molti altri ricercatori con ottimi risultati.

Senza entrare nei dettagli della trattazione teorica, per la quale rimandiamo all'originale, ei limiteremo qui a trascrivere le formule essenziali per il calcolo, con quei chiarimenti atti alla comprensione dei simboli.

Se $t_{o}, \lambda_{o}, \varphi_{o}, h_{o}$ sono i valori approssimati delle 4 incognite (assunti in un modo qualunque che diremo tra breve) il procedimento tende a calcolare le piccole correzioni da apportare ad essi: $\delta t_{o}, \delta \lambda_{o}, \delta \varphi_{o}, \delta h_{o}$. A tale scopo occorre risolvere il seguente sistema di $n$ equazioni $d i$ condizione (tante quante sono le stazioni utilizzate):

$$
t n=f\left(\lambda_{o}, \varphi_{o}, h_{o}\right)+\frac{\lambda f}{\partial \lambda_{o}} \delta \lambda_{o}+\frac{\partial f}{\partial \varphi_{o}} \delta \varphi_{o}+\frac{i f f}{\partial h_{o}} \delta h_{o}
$$

che si può mettere anche sotto la forma

$$
\begin{gathered}
\partial \mathrm{t}_{o}-\cos \varphi_{o} \sin \alpha \frac{\partial f}{\partial \Delta_{n}} \delta \lambda_{o}-\cos \alpha \frac{\partial f}{\partial \Delta_{n}} \delta \tilde{o}_{0}=\frac{\partial f}{\partial h_{o}} \delta \mathbf{h}_{0}- \\
=T_{n}-\left[t_{o}+f\left(\lambda_{o} \varphi_{o} h_{o}\right)\right]
\end{gathered}
$$

Ricordiamo il significato dei simboli che compaiono nella $[1]=\left[1^{\prime}\right]$ : $t_{n}$ è il tempo di propagazione delle prime onde

$f\left(\lambda_{o}, \varphi_{o}, h_{o}\right)$ è il tempo di propagazione delle prime onde, corrispondente alla distanza epicentrale $\Delta$ della stazione considerata, distanza calcolata in base all'epicentro provvisorio.

$\frac{\partial f}{\partial \lambda_{o}} \mathrm{e} \frac{\partial f}{\partial \varphi_{o}} \mathrm{e} \frac{\partial f}{\partial h_{o}}$ rappresentano le variazioni del tempo di propagazione delle $P$ al variare rispettivamente di $\lambda_{o}, \varphi_{o}, h_{o}$.

$\alpha$ è l'azimut di ciascuna stazione rispetto all'epicentro. Per una formula di trigonometria sferica si ha che

$$
\sin \alpha=\frac{\cos \varphi \sin \left(\lambda-\lambda_{o}\right)}{\sin \Delta}
$$


$\frac{\partial f}{\partial \Delta_{n}}$ è, per 7 costante, l'incremento che subisce ogni tempo di tragitto $\frac{\partial f}{\partial h_{0}}$ è, per $\Delta$ costante, l'incremento che subisce ogni tempo di tragitto quando $h$ viene incrementata di una unità.

$T_{n}$ è il tempo d'arrivo delle onde $P$ registrate nelle varie stazioni.

$t_{o}$ è il tempo origine prescelto.

Per il calcolo delle $\Delta$, vale la relazione seguente (che si ricava dalla trigonometria sferica):

$$
\cos \Delta=\sin \varphi_{0} \sin \varphi+\cos \varphi_{0} \cos \varphi \cos \left(\lambda-\lambda_{0}\right)
$$

essendo $\varphi$ e $\lambda$ le coordinate geografiche della stazione prescelta.

Ciò premesso possiamo procedere al calcolo di quegli elementi che sono necessari per giungere alla [1'].

I valori ipocentrali di partenza $\left(\varphi_{o}, \lambda_{o}, h_{o}\right)$ li assumeremo facendo una media tra quelli dati da alcuni dei più importanti centri di raccolta del mondo. Per quanto riguarda la profondità ipocentrale, trattandosi a prima vista di un terremoto superficiale, abbiamo assunto per comodità il valore

$$
h_{0}=5 \mathrm{~km}
$$

il che ei consente di utilizzare la dromocrona calcolata per l'Italia centrale ad una profondità di $5 \mathrm{~km}$. I termini $\frac{\partial f}{\partial \hbar_{o}}$ li calcoleremo scegliendo come seconda dromocrona quella calcolata per l'Italia settentrionale ad una profondità media di

$$
h_{0}=17 \mathrm{~km} \text {. }
$$

Partiremo pertanto dal seguente ipocentro provvisorio

$$
\left\{\begin{array}{l}
\varphi_{0}=46^{\circ} 28^{\prime} 30^{\prime \prime} \mathrm{N} \\
\lambda_{0}=13^{\circ} 00^{\prime} 20^{\prime \prime} \mathrm{E} \\
h_{0}=5 \mathrm{~km} .
\end{array}\right.
$$

Calcoliamo, con la [2], le distanze epicentrali di tutte le stazioni utilizzabili, le quali risultano in numero di 12 . Infatti, dovendo servirci delle $P_{n}$, abbiamo dovuto escludere da questi calcoli le stazioni troppo vicine. 


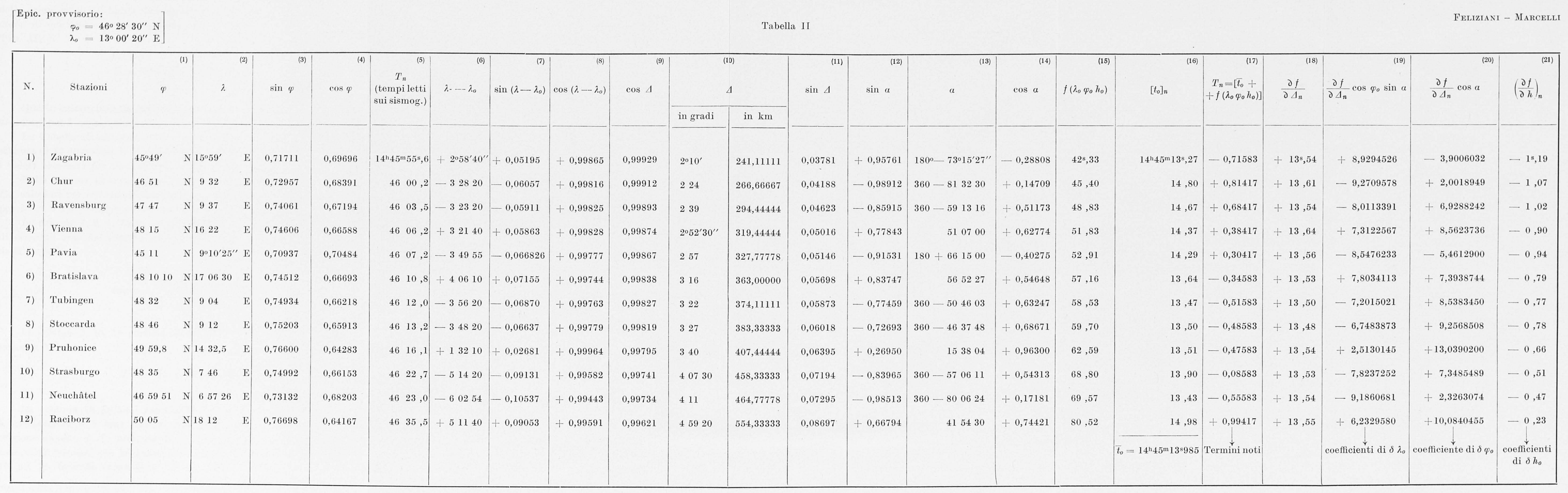


Per trovare il valore provvisorio del tempo origine abbiamo fatto la media delle differenze tra $\mathrm{i}$ tempi d'arrivo $T_{n}$ (V. col. 5 della Tab. II) delle prime onde e i tempi di tragitto $f\left(\lambda_{o} \varphi_{o} h_{o}\right)$ calcolati dalla dromocrona di cui sopra (per $h=5 \mathrm{~km}$ ). Ne è risultato: (v. col. 16 della Tab. II).

$$
\overline{t_{o}}=14^{\mathrm{h}} 45^{\mathrm{m}} 13^{\mathrm{s}}, 98583
$$

Questo valore, insieme con le [3], costituisce i 4 elementi di partenza necessari per l'impostazione della [1] o [1'].

La Tabella II raccoglie tutti i dati necessari alla impostazione del sistema di 12 equazioni in 4 incognite. Le colonne $3,4,6,8,9$, mostrano i valori necessari per giungere alle varie $\Delta$ della colonna 10 .

Nella colonna 15 compaiono i tempi di propagazione $f\left(\lambda_{o} \varphi_{o} h_{o}\right)$ delle $P_{n}$, calcolati per interpolazione (dalla dromocrona di Di Filippo-Marcelli per $h=5 \mathrm{~km}$ ) relativamente ad ogni distanza epicentrale.

Le colonne 4, 6, 7, 11 servono per calcolare l'azimut $\alpha$ (col. 12, 13) di ciascuna stazione rispetto all'epicentro, che come s'è visto, è dato dalla formula.

$$
\sin a=\frac{\cos \varphi}{\sin \left(\lambda-\lambda_{0}\right)}
$$

Le colonne 5, 15, 16 portano al calcolo dei termini noti del sistema (colonna 17):

$$
T_{n}-\left[\bar{t}_{o}+f\left(\lambda_{o} \varphi_{o} h_{o}\right)\right]
$$

La colonna $18\left[\partial f / \partial A_{n}\right]$ rappresenta l'incremento che subisce (per $h$ costantemente $5 \mathrm{~km}$ ) ciascun tempo di tragitto relativo ad ogni stazione quando la $\Delta_{n}$ aumenta di 1 grado: ed anche questi incrementi sono stati calcolati con la dromocrona per $h-5 \mathrm{~km}$. Infine le colonne $19,20,21$, tenendo conto della col. 14 , danno rispettivamente i coefficienti delle 3 incognite $\delta \lambda_{o}$, ò $\varsigma_{o}, \delta h_{o}$. I valori $\left(\frac{\partial f}{\partial h}\right)$ dell'ultima colonna (21) rappresentano l'incremento che subisce il tempo $f\left(\lambda_{0} \varphi_{o} h_{o}\right)$ quando, mantenendo costanti le $\Delta_{n}$, si passa dalla dromocrona di $h=5 \mathrm{~km}$ a quella di Caloi per $h=17 \mathrm{~km}$. 
Tenendo presente che i coefficienti della IV incognita oto sono tutti uguali ad 1, possiamo impiantare numericamente il nostro sistema [1'] di 12 equazioni nelle $t$ incognite nel modo seguente:
1) Kagabria
$\mathrm{jt}_{\mathrm{o}}-8,9294526 \mathrm{j}_{\mathrm{k}}+3,9006032$
2) Chur
" $+9,2709578$ " 2,0018949
3) Ravensburg " $+8,0113391 "-6,9288242$
) $-1,07$
$-0,81417=0$
4) Vienna
" - 7,3122567 " 8,5623736
$\gg-1,02$
" $-0,68417=0$
5) Pavia
" $+8,5476233 "+5,4612900$
" - $0,90 "-0,38417=0$
6) Bratislava
" $-7,8034113 " 7-7,3938744$
" $-0,94$ " $-0,30417=0$
7) Tubingen
" $+7,2015021 "-8,5383450$
" - $0,79 "+0,34583=0$
8) Stoccarda
" - 0,77
" $+0,51583=0$
9) Pruhonice
" $+6,7483873$ » $-9,2568508$
" $-2,5130145$ " $-13,0390200$
$-0,78 \cdots+0,48$ อ $13=0$
10) Strasburgo
11) Neuchâtel
" $+7,8237252 "-7,3485489$
" $-0,66 "+0,47583=0$
" $+9,1860681$ " $-2,3263074$
" $-0,51 "+0,08583=0$
12) Raciborz
" $-6,2329580$ » $-10,0840455$
" $-0,47 "+0,55583=0$
" $-0,23$ " $-0,99417=0$

Le equazioni del sistema [5] sono le equazioni normali di equazioni più generiche del tipo

$$
a_{\imath} x+b_{i} y+c_{i} z+d_{i} k+l_{i}=v_{i}
$$

Nelle [6] i $v_{i}$ rappresentano i residui dovuti agli errori d'osservazione. Tali residui ovviamente devono essere i più piccoli possibile. Essi verramno dati dalle [5] stesse quando, una volta risolto il sistema, si sostituiscono i valori delle incognite in ciascuna di esse.

Le [6] vanno risolte con il metodo dei minimi quadrati, e si sa dalla teoria che tale metolo conduce a risolvere il sistema di equazioni normali secondo lo sehema seguente dovuto a Gauss:

$$
\begin{array}{r}
{[a a] x+[a b] y+[a c] z+[a d] k+[a l]=0} \\
{[b b .1] y+[b c .1] z+[b d .1] k+[b l .1]=0} \\
{[c c .2] z+[c l .2] k+[c l .2]=0} \\
{[d l .3] k+[d l .3]=0}
\end{array}
$$

Senza dilungarei sul significato dei coefficienti delle [7], che riteniamo noto, diremo che, a calcoli fatti, l'analogo del [7] è risultato per noi il seguente:

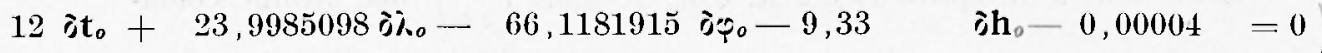

$$
\begin{aligned}
& +657,4593816 "+83,1183519 "-0,1199215 "-4,1390037=0 \\
& +339,9403249 "-8,8492609 "+1,7523663=0
\end{aligned}
$$


La soluzione del sistema [8] ci ha dato, per le nostre incognite, i seguenti valori:

$$
\delta h_{o}=+0,0760812
$$

Poichè nei nostri calcoli abbiamo variato la profondità ipocentrale tra i 5 e i $17 \mathrm{~km}$, ne segue che il $\delta h_{0}$ trovato rappresenta la frazione di $(17-5) \mathrm{km}$, vale a dire

$$
\delta h_{o}=+0,9129744 \mathrm{~km}
$$

Per le altre incognite abbiamo trovato

$$
\begin{aligned}
& \delta \varphi_{o}=-0^{\circ}, 0031744=-0^{\circ} 00^{\prime} 11^{\prime \prime}, 428 \\
& \delta \lambda_{o}=+0^{\circ}, 0067106=+0^{\circ} 00^{\prime} 24^{\prime \prime}, 158 \\
& \delta t_{o}=+0^{\mathrm{s}}, 0282456
\end{aligned}
$$

La sostituzione di questi valori nelle [5] dà i residui relativi: essi a calcoli fatti, sono i seguenti:

$$
\begin{array}{llc} 
& & v_{i} \\
\text { 1) Zagabria : } & +0,5812349 \\
\text { 2) Chur : } & -0,7987628 \\
\text { 3) Ravensburg : } & -0,6577714 \\
\text { 4) Vienna : } & -0,4462867 \\
\text { 5) Pavia : } & -0,3074173 \\
\text { 6) Bratislava : } & +0,2850770 \\
\text { 7) Tubingen : } & +0,5609236 \\
\text { 8) Stoccarda : } & +0,5294029 \\
\text { 9) Pruhonice : } & +0,4783893 \\
\text { 10) Strasburgo : } & +0,1511033 \\
\text { 11) Neuchâtel } & : & +0,6173460 \\
\text { 12) Raciborz } & : & -0,9932392 .
\end{array}
$$

Per l'esattezza dei calcoli deve risultare

$$
[11.4]=[v v]
$$

ed infatti abbiamo:

$$
\begin{aligned}
& {[11.4]=+3,9976971} \\
& {[v v]=+3,9976968 .}
\end{aligned}
$$


Poichè i risultati ottenuti sono soddisfacenti, procediamo anche al calcolo degli errori: tale calcolo è laboriosissimo, ma noi riportiamo soltanto i valori finali che sono i seguenti:

$$
\begin{aligned}
& \varepsilon \quad= \pm \sqrt{\frac{[11.4]}{n-4}}= \pm 0,7069032 \\
& m_{\delta t_{o}}= \pm \frac{\varepsilon}{\sqrt{[a a .3]}}= \pm 0^{\mathrm{s}, 8960966} \\
& m_{\delta \lambda_{o}}= \pm \frac{\varepsilon}{\sqrt{[b b .3]}}= \pm 0^{\circ}, 0281343= \pm 0^{\circ} 1^{\prime} 41^{\prime \prime}, 283480 \\
& m_{\delta \varphi_{\circ}}= \pm \frac{\varepsilon}{\sqrt{[c c .3]}}= \pm 0^{\circ}, 0450479= \pm 0^{\circ} 2^{\prime} 42^{\prime \prime}, 17244 \\
& m_{\delta h_{o}}= \pm \frac{\varepsilon}{\sqrt{[d d .3]}}= \pm 0,9084981= \pm 10,9019772 \mathrm{~km} .
\end{aligned}
$$

Sicchè l'ipocentro del nostro terremoto, tenuto conto dei dati di partenza [3] e [4], delle relative correzioni trovate [9], e degli errori trovati $[10]$, diventa:

$$
\begin{aligned}
& \Phi_{o}=\left[\varphi_{o}+\delta \varphi_{o}\right] \pm m_{\delta \varphi_{o}}=\left[46^{\circ} 28^{\prime} 18^{\prime \prime}, 572 \pm 02^{\prime} 42^{\prime \prime}, 172\right] \text { Nord } \\
& A_{o}=\left[\lambda_{o}+\delta \lambda_{o}\right] \pm m_{\delta \lambda_{o}}=\left[13^{\circ} 00^{\prime} 44^{\prime \prime}, 158 \pm 01^{\prime} 41^{\prime \prime}, 283\right] \text { Est } \\
& H_{o}=\left[t_{o}+\delta t_{o}\right] \pm m_{\delta t_{o}}=\left[14^{\mathrm{n}} 45^{\mathrm{m}} 14^{\mathrm{s}}, 014 \pm 0 \mathrm{~s}, 896\right] \\
& h=\left[h_{o}+\delta h_{o}\right] \pm m_{\delta h_{o}}=\mathrm{km}[5,913 \pm 10,902]
\end{aligned}
$$

\section{c) Osservazioni.}

L'epicentro del nostro terremoto risulta quindi ubicato nelle immediate vicinanze di Zuglio (località che ha infatti determinato la massima intensità macrosismica), quasi al punto d'incontro della linea di Sauris con la faglia, di direzione S-W, che dalla estremità di questa linea si prolunga fin quasi ad incontrare la linea parallela del medio Tagliamento più a Sud (v. schema $2 b$ ); esso dista $3-4 \mathrm{~km}$ dalla fascia di dislocazione But-Chiar'sò e $5-6 \mathbf{~ k m}$ dall'epicentro dell'altro forte terremoto del 27 Marzo 1928 che si trova invece ad oriente di detta fascia.

La profondità ipocentrale risulta molto piccola; il terremoto è da ritenersi superficiale o, al massimo, contenuto ampiamente entro i limiti dello strato del granito. 
Natura DELLA SCOSSA ALL'IPOCENTRO E DISCUSSIONE DEI RISUltati.

Nella Tabella III riportiamo i primi impulsi registrati in molte delle stazioni da noi esaminate. Esse risultano in numero maggiore di

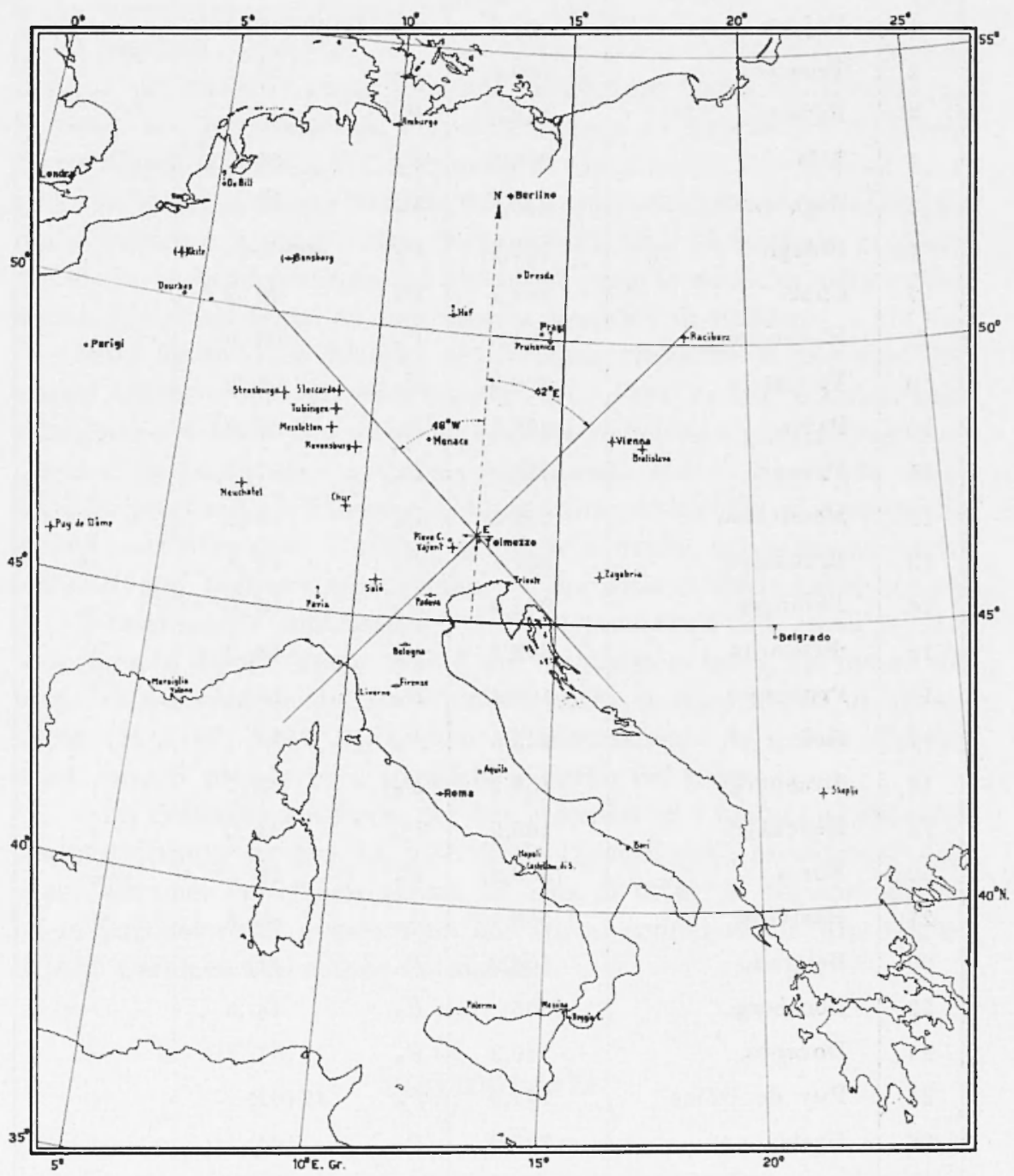

Fig. 4

quelle usate per i calcoli precedenti, perchè in questo esame abbiamo potuto includere anche quelle stazioni scartate per motivi vari (o perchè 
Tabella III

\begin{tabular}{|c|c|c|c|c|c|}
\hline $\mathrm{N}$ & Stazioni & $\Delta($ in $\mathrm{km})$ & \multicolumn{2}{|c|}{$\begin{array}{l}\text { Ten pi d'arrivo } \\
\text { delle prime onde }\end{array}$} & $\begin{array}{l}\text { Natura del } \\
1 \text { impulso }\end{array}$ \\
\hline 1 & Vajont & 55,5 & $P_{g}$ & $14^{h} 45^{m} 26^{s}, 4$ & + \\
\hline 2 & Trieste & 109,8 & $P_{g}$ & 35,2 & $\ldots$ \\
\hline 3 & Padova & 146,8 & $P_{g}$ & 42,1 & $\ldots$ \\
\hline 4 & Salò & 213,7 & $P_{n}$ & 51,7 & $\therefore$ \\
\hline 5 & Zagabria & 240,0 & $P_{n}$ & 55,6 & + \\
\hline 6 & Bologna & 257,1 & $P_{g}$ & $46^{\mathrm{m}} 01 \mathrm{~s}, 8$ & - \\
\hline 7 & Chur & 268,1 & $P_{n}$ & 00,2 & + \\
\hline 8 & Ravensburg & 294,8 & $P_{n}$ & 03,5 & + \\
\hline 9 & Vienna & 320,3 & $P_{n}$ & 06,2 & + \\
\hline 10 & Pavia & 329,7 & $P_{n}$ & 07,2 & $?$ \\
\hline 11 & Firenze & 330,1 & $P_{n}$ & $(07)$ & - \\
\hline 12 & Messtetten & 359,5 & $P_{n}$ & 10,5 & + \\
\hline 13 & Bratislava & 361,6 & $P_{n}$ & 10,8 & + \\
\hline 14 & Tubingen & 374,9 & $P_{n}$ & 12,0 & + \\
\hline 15 & Stoccarda & 383,3 & $P_{n}$ & 13,2 & + \\
\hline 16 & Pruhonice & 408,4 & $P_{n}$ & 16,1 & $?$ \\
\hline 17 & Hof & 435,6 & $P_{n}$ & $(20)$ & $\ldots$ \\
\hline 18 & Strasburgo & 458,2 & $P_{n}$ & 22,7 & -4 \\
\hline 19 & Neuchâtel & 465,0 & $P_{n}$ & 23,0 & $\therefore-$ \\
\hline 20 & Roma & $(509,2)$ & $P_{n}$ & 26 & -- \\
\hline 21 & Raciborz & 555,1 & $P_{n}$ & 35,5 & $\frac{1}{1}$ \\
\hline 22 & Belgrado & 606,0 & $P_{n}$ & (41) & $?$ \\
\hline 23 & Bensberg & $(655)$ & $P_{n}$ & 48,5 & $(-)$ \\
\hline 24 & Dourbes & 740,3 & $P_{n}$ & $(57,2)$ & - \\
\hline 25 & Puy de Dôme & 777,3 & $P_{n}$ & $47(02)$ & $\therefore$ \\
\hline 26 & Uccle & 795,9 & $P_{n}$ & $(04)$ & $(j)$ \\
\hline 27 & Amburgo & 807,6 & $P_{n}$ & $05, \mathrm{I}$ & $(-)$ \\
\hline 28 & De Bilt & 843,9 & $P_{n}$ & (I0) & $?$ \\
\hline 29 & Skopije & $(900)$ & $P_{n}$ & 10,0 & + \\
\hline
\end{tabular}


troppo vicine, o perchè prive di correzione del tempo etc....). La Fig. 4 mostra la distribuzione dei segni: ne risulta uno schema di frattura verticale: trattandosi di terremoto con ipocentro praticamente in superficie, la frattura si deve ritenere causata da una coppia orizzontale di forze applicata nel fuoco sismico; l'orientamento delle linee nodali è con buona approssimazione $48^{\circ} \mathrm{W}$ e $42^{\circ} \mathrm{E}$.

I risultati conseguiti in quest'ultima parte della nostra ricerca, portano un ulteriore contributo alle conclusioni tratte da Di FilippoPeronaci nel loro studio su un certo numero di terremoti interessanti l'arco Alpino orientale. I 7 terremoti da essi studiati (tra i quali compare quello del 27 Marzo 1928 da noi ripetutamente citato, con epicentro molto prossimo al nostro) sono stati originati tutti da fratture, e questo rientra in un quadro ancora più vasto nel quale la sismicità della catena alpina è caratterizzata da uno schema generale di fratture.

Sulla figura dello schema $2 b$ ) abbiamo riportato la posizione del nostro epicentro con gli orientamenti delle linee nodali: è abbastanza sintomatico il fatto che tali orientamenti coincidano praticamente con quelli della faglia " $f a$ " e dell'accavallamento che si diparte da detta faglia in prossimità dell'epicentro, in posizione ortogonale ad essa: questo secondo orientamento è altresi parallelo a quello dei numerosi strati inclinati che tagliano trasversalmente la zona a Sud dell'epicentro.

E interessante confrontare i risultati precedenti con quelli relativi al terremoto del 27 Marzo 1928 il cui epicentro si trova nel mezzo del fascio di dislocazione del But-Chiarsó: anche le linee nodali di questo sisma $\left(55^{\circ}, 5 \mathrm{~W} ; 34^{\circ}, 5 \mathrm{E} ;\right)$ seguono l'orientamento di queste dislocazioni, e sono press'a poco parallele a quelle del nostro.

Data l'estrema vicinanza dei due epicentri ed i molteplici elementi comuni, diventa sempre più attendibile l'ipotesi della esistenza di una causa comune: l'orogenesi ancora in atto di tutta la regione alpina, ed in particolare di questa zona che sta ai confini tra le Alpi Giulie, le Alpi Carniche e la catena Paleozoica.

\section{BIBIJIOGRAFIA}

Feliziani P., Marcelli L., Il terremolo di Tolmezzo del 26 Aprile 1959. I parte: Tenti secoli di storia sismica della Regione Carnica e dell'Italia Nord-Orientale. "Annali di Geofisica ", XVIII, 3, (1965).

SELLI R., Cenni stratigrafici e tettonici sulle Alpi Carniche e Giulie Occidentali. "Bollettino della Società Geologica Italiana ", LXXXIII, fasc. 3 (196t). 
Gortani M., Il terremoto del 27. Larzo 1928 nelle Prealpi dell'Arzino (Friuli). "L'Universo ", IX, 12, (1928).

Di Filippo D., Peronaci F., Terremoti di fraltura e relazioni con la tettonica nelle Alpi Orientali. "Annali di Geofisica ", XV, 2-3, (1962).

Caloi P., Peronaci F., Il terremoto del Turkestan del 2 Novembre 1946. "Annali di Geofisica ", 1, 2 (1948).

Di Filippo D. Marceldi L., Dromocrone per terremoti vicini e velocità delle onde nell'Italia Centrale. "Annali di Creofisica "V, 2, (1952).

CaLor P., Tempi di tragitto per terremoti ad origine vicina. "La Ricerca Scientifica " $\mathbf{X}, 5,(1939)$. 